\title{
Thank You, Referees!
}

Every professional journal is completely dependent on the enthusiasm, wisdom, and hard work of its referees, who review each article in detail. Dreaming wishes to express its gratitude to the following who have served as referees over the past two years:

$\begin{array}{ll}\text { Abramovitch, Henry } & \text { Hollister, Robin } \\ \text { Antrobus, John } & \text { Holms, Mark } \\ \text { Baylor, George } & \text { Hufford, D.J. } \\ \text { Belicki, Kathryn } & \text { Hughes, Scott } \\ \text { Berry, Patricia } & \text { Hunt, Harry } \\ \text { Blagrove, Mark } & \text { Kahn, David } \\ \text { Bograzan, Fariba } & \text { Kennard, L.R. } \\ \text { Bulkeley, Kelly } & \text { Kilroe, Patricia } \\ \text { Bursik, Kris } & \text { King, Johanna } \\ \text { Bynum, Edward Bruce } & \text { Korde, Sharish } \\ \text { Campbell, Jean } & \text { Koulack, David } \\ \text { Cartwright, Rosalind } & \text { Krakow, Barry } \\ \text { Cohen, David } & \text { Kramer, Milton } \\ \text { Cuddy, Marion } & \text { Krippner, Stanley } \\ \text { DeGarcia, Don } & \text { Kugler, Paul } \\ \text { Delaney, Gayle } & \text { LaBerge, Stephen } \\ \text { Dombeck, Mary } & \text { Lederbogen, Sibylle } \\ \text { Domhoff, William } & \text { Levin, Ross } \\ \text { Donderi, D.C. } & \text { McCormick, Maureen } \\ \text { Doniger, Wendy } & \text { McGill, David } \\ \text { Edgar, Iain } & \text { Meir Faber, Barbara } \\ \text { Evans, Bradford } & \text { Nielsen, Tore } \\ \text { Fosse, Roar } & \text { Pace-Schott, Edward } \\ \text { Foulkes, David } & \text { Pagel, Jim } \\ \text { Gackenback, Jayne } & \text { Palombo, Stanley } \\ \text { Greenberg, Ramon } & \text { Parker, Jennifer } \\ \text { Halliday, Gordon } & \text { Poe, Richard } \\ \text { Harrison, Robert } & \text { Porte, Helene } \\ \text { Hartmann, Ernest } & \text { Radtke, H.L. } \\ \text { Heynick, Frank } & \text { Rainville, Raymond } \\ \text { Hoffman, Robert } & \text { Revonsuo, Antti } \\ & \end{array}$


Roese, Neal

Rosner, Rachel

Rupprecht, Carol

Russo, Richard

Schmidt, Dennis

Schredl, Michael

Sherwood, Simon

Siegel, Alan

Stepakoff, Shanee
Stickgold, Robert

Strauch, Inge

Terwee, Sybe

Van de Castle, Robert

White, Sarah

White-Lewis, Jane

O'Toole, Win

Zadra, Antonio

Zborowski, Michael 